Proceedings of SALT 24: 473-493, 2014

\title{
Generalized focus intervention *
}

\author{
Haoze Li \\ Jess Law \\ The Chinese Univ. of Hong Kong Rutgers, the State Univ. of New Jersey
}

\begin{abstract}
In a recent article (Li \& Law 2014), we argue that focus intervention is a manifestation of inappropriate quantificational domains of focus-sensitive operators, resulting from the interaction of focus alternatives and ordinary alternatives. The theory predicts that expressions introducing ordinary alternatives should all be subject to focus intervention, just as interrogative $w h$-phrases are. This paper bears out the prediction with non-interrogative $w h$-phrases and disjunctive phrases.
\end{abstract}

Keywords: focus intervention, alternative semantics, non-interrogative wh, disjunction

\section{Introduction}

Since Beck's (2006) seminal paper, alternative semantics has been widely accepted as a framework for studying focus intervention. Subsequent studies that follow this tradition include Beck \& Kim 2006, Cable 2010 and Kotek \& Erlewine to appear. We agree with these studies that alternative semantics can help us gain important insights into focus intervention. However, following Li \& Law 2014, we take a departure from the framework developed in Beck 2006 and defend a quantificational domain approach to focus intervention. This approach synthesizes two branches of alternative semantics, namely Hamblin's (1973) original alternative semantics and Rooth's (1992) focus semantics. We demonstrate that this approach has greater explanatory power because of its precision and generality in accounting for focus intervention.

A typical case of focus intervention can be seen in the Mandarin example (1a), in which a focus-sensitive operator precedes both a focused phrase and a wh-phrase. ${ }^{1}$

* Different parts of this work have been presented at NELS-44 (University of Connecticut) and GLOW-37 (CRISSP, Brussels). We thank the anonymous reviewers and the audiences there for their comments and criticisms. We are also grateful to Angelika Kratzer, Billy Xu, Ezra Keshet, Jo-Wang Lin, Roger Schwarzschild, Satoshi Tomioka, Simon Charlow, Veneeta Dayal and Yi-Hsun Chen for their generous comments on various versions of this work. We gratefully acknowledge Sarah Hansen, Vartan Haghverdi and Simon Charlow for their help with the English data and judgments. Special thanks go to Mia Wiegand for her editorial help. As always, none of these people should be held responsible for any inadequacies in this article.

1 Throughout the paper, focus-sensitive operators are set in italics and $w h$-phrases (and their semantic

(C)2014 Li \& Law 
We have shown in Li \& Law 2014 that focus intervention is absent in the minimally different (1b), in which the focus-sensitive operator takes the wh-phrase as its associate.
a. * Ta zhi yaoqing-le Libai $F$ chuxi shenme huodong? he only invite-Asp Libai attend what activity
'What is the activity $\mathrm{x}$ such that he only invited Libai $F$ to attend $\mathrm{x}$ ?'
b. Libai zhi chuxi-le shenme huodong? Libai only attend-Asp what activity
'What is the activity $\mathrm{x}$ such that Libai only attended $\mathrm{x}$ ?'

Based on the contrast between (1a) and (1b), we have argued against the view (due to Beck 1996, 2006) that focus intervention is triggered merely by the presence of focus-sensitive operators intervening between $w h$-phrases and their operators. Instead, we argued that the phenomenon is related to the quantificational structure of focus-sensitive operators, which is determined by what occurs in conjunction with the $w h$-phrases in the scope of the focus-sensitive operators.

We have assumed that a $w h$-phrase and a focus phrase denote sets of alternatives along different dimensions. Specifically, the former denotes a set of alternatives as its ordinary-semantic value (Hamblin 1973), and the latter denotes a set of alternatives as its focus-semantic value (Rooth 1992). These two types of alternatives are referred to as ordinary alternatives and focus alternatives, respectively. When ordinary alternatives and focus alternatives appear in the scope of a focus-sensitive operator, as in the case of (1a), they interact and give rise to a focus-semantic value that denotes a set of sets of alternatives. This focus-semantic value cannot serve as the quantificational domain of the focus-sensitive operator, as it would result in composition failure and hence focus intervention. On the contrary, if a focussensitive operator only scopes over a $w h$-phrase (or a focused phrase, which we will ignore here), as in (1b), no such interaction would result, hence no focus intervention is triggered. The ability to account for this contrast without ad hoc assumptions makes the quantificational domain approach a more precise, and hence attractive, theory for focus intervention.

Their approach makes possible the following focus intervention configuration:

$$
\text { *[...focus-sensitive operator [focus alternatives ... ordinary alternatives ...]] }
$$

This configuration leads to the second merit of the quantificational domain approach: generality. Note that there is no mention of interrogative wh-phrases in (2). All that

parallels) are set in boldface; focused phrases are immediately followed by a subscript $F$, which can be understood as a focus feature (Rooth 1985, 1992) or a focus index (Kratzer 1991).

In addition, the following abbreviation is used in the gloss: Asp = aspectual marker. 
matters is for ordinary alternatives to co-occur with focus alternatives in the scope of a focus-sensitive operator. What gives rise to ordinary alternatives and focus alternatives is independent of the theory of focus intervention.

Luckily, there are independent studies that we can draw on to decide what expressions give rise to what alternatives. In particular, focus alternatives are triggered by focused phrases, as first argued by Rooth $(1985,1992)$. Ordinary alternatives can be introduced by a host of expressions, among which are interrogative $w h$-phrases (Hamblin 1973), non-interrogative $w h$-phrases (Kratzer \& Shimoyama 2002, Shimoyama 2006) and disjunctive phrases (Aloni 2003, Simons 2005, Alonso-Ovalle 2006). If the quantificational domain approach to focus intervention is on the right track, the configuration in (2) should apply not only to sentences with interrogative wh-phrases, but also to sentences with non-interrogative $w h$-phrases or disjunctive phrases. One goal of this paper is to show that this prediction is indeed correct.

This paper is organized as follows: we offer a brief introduction to the quantificational domain approach to focus intervention in section 2 . It is followed by a discussion of focus intervention with non-interrogative wh-phrases in section 3 , disjunctive phrases in declarative sentences in section 4.1, and disjunctive phrases in questions in section 4.2. Section 5 elaborates on our assumption regarding the availability of focus indices. Section 6 concludes the paper.

\section{The quantificational domain approach to focus intervention}

The quantificational domain approach asserts that focus intervention is a consequence of illicit quantificational domains of focus-sensitive operators, resulting from the interaction of sets of alternatives along different dimensions. This section describes how this approach accounts for the contrast in (1a) and (1b). We begin with the case of focus intervention (i.e., 1a), whose LF is shown below:

$$
\begin{aligned}
& \text { ?* [IP Ta [VP2 zhi [VP1 yaoqing-le Libai } \left.\left.{ }_{F 1} \text { chuxi shenme huodong] }\right]\right] \text { ? } \\
& \text { he only invite-Asp Libai attend what activity } \\
& \text { 'What is the activity x such that he only invited Libai }{ }_{F} \text { to attend x?' }
\end{aligned}
$$

Following Hamblin (1973), in Li \& Law 2014 we hold a simplistic view of the denotation of $w h$-phrases, taking them to denote sets of alternatives as their ordinarysemantic values (contra Beck 2006). Along these lines, the denotation of shenme huodong 'what activity' is shown in (4a). We further assume that $w h$-phrases, unlike ordinary focused phrases, do not bear any focus index (see section 5), hence do not trigger the use of Kratzer's (1991) focus assignment function, faithfully represented as $h$ in this article. The ordinary-semantic value of VP1 is derived by applying the denotation of the verb to that of the $w h$-phrase pointwisely. We refer the reader to Li \& Law 2014 and Yatsushiro 2009:152 for the variant of pointwise functional 
application the quantificational domain approach adopts (see Hamblin 1973 for the original idea). The result is given in (4b).

a. $\llbracket$ shenme huodong $\rrbracket^{g}=\{$ John's birthday party, Mary's wedding, ... $\}$

b. $\llbracket \mathrm{VP} 1 \rrbracket^{g}=\left\{\lambda y . \lambda w\right.$. invited-to-attend ${ }_{w}(y$, Libai, $x) \mid x \in \llbracket$ shenme huodong $\left.\rrbracket^{g}\right\}$

When $z h i$ 'only' is applied, its semantics requires that $\llbracket \mathrm{VP} 1 \rrbracket^{g}$ (i.e., the ordinarysemantic value of VP1) be the only predicate that yields a true proposition after applying to the subject, among all the alternatives denoted by $\llbracket \mathrm{VP} 1 \rrbracket^{f}$ (i.e., the focus-semantic value of VP1), as indicated by (5). We can say that $\llbracket \mathrm{VP} 1 \rrbracket^{f}$ provides the quantificational domain for the focus-sensitive operator $z h i$.

$$
\llbracket z h i \mathrm{VP} 1 \rrbracket^{g}=\lambda y . \lambda w . \forall P \in \llbracket \mathrm{VP} 1 \rrbracket^{f}\left[P_{w}(y) \rightarrow \llbracket \mathrm{VP} 1 \rrbracket^{g}(y) \subseteq P(y)\right]
$$

To derive $\llbracket \mathrm{VP} 1 \rrbracket^{f}$, Li \& Law (2014) make use of the designated assignment function $h$ devised by Kratzer (1991). Specifically, $h$ computes $\llbracket$ VP1 $\rrbracket^{g, h}$ (i.e., the secondary value of VP1), relativized to different assignments to the focus index borne by the focused phrase, i.e., Libai in this case. The result is (6a). Assuming that $\mathrm{H}$ is the set of designated assignments, we obtain a set of the secondary values of VP1, i.e., $\llbracket \mathrm{VP} 1 \rrbracket^{f}$, as in (6b).

$$
\begin{aligned}
& \text { a. } \llbracket \mathrm{VP} 1 \rrbracket^{g, h}=\left\{\lambda y \cdot \lambda w \text {. invited-to-attend }{ }_{w}(y, h(1), x) \mid x \in \llbracket \text { shenme huodong } \rrbracket^{g}\right\} \\
& \text { b. } \llbracket \mathrm{VP} 1 \rrbracket^{f}=\left\{\llbracket \mathrm{VP} 1 \rrbracket^{g, h} \mid h \in \mathrm{H}\right\} \\
& =\left\{\left\{\lambda y . \lambda w \text {. invited-to-attend }(y, h(1), x) \mid x \in \llbracket \text { shenme huodong } \rrbracket^{g}\right\} \mid h \in \mathrm{H}\right\}
\end{aligned}
$$

Returning to the denotation in (5), we see that the quantificational domain of $z h i$ is illicit: $\llbracket \mathrm{VP} 1 \rrbracket^{f}$ is a set of sets of properties, but what zhi quantifies over are properties. Since no property can be a member of a set of sets of properties, $z h i$ cannot apply to VP1 and the derivation fails, giving rise to focus intervention.

The quantificational domain approach also explains why focus intervention is absent when a focus-sensitive operator is associated with a wh-phrase. The LF structure of (1b) is given in (7), whose semantic composition is enunciated in (8). Since VP1 does not contain any focused phrase, we follow Kratzer 1991 and take its secondary-semantic value to be simply identical to its ordinary-semantic value. Crucially, we assume that the focus-sensitive operator takes $\llbracket \mathrm{VP} 1 \rrbracket^{g, h}$ as its quantificational domain and applies to $\llbracket \mathrm{VP} 1 \rrbracket^{g}$ in a pointwise manner. As shown in (8b), «VP1 $\rrbracket^{g, h}$ denotes a set of properties, constituting a well-formed quantificational domain for $z h i$, which quantifies over properties (we use ' $\approx$ ' to introduce simplified, approximate denotation). Therefore, no focus intervention is triggered. The result yields a set of propositions, as shown in (8c).

$$
\begin{gathered}
[I P \text { Libai }[V P 2 \text { zhi [VP1 chuxi-le shenme huodong }]]] ? \\
\text { Libai only attend-Asp what activity }
\end{gathered}
$$

'What was the activity $\mathrm{x}$ such that Libai attended nothing other than $\mathrm{x}$ ?' 
Generalized focus intervention

a. $\llbracket \mathrm{VP} 1 \rrbracket^{g}=\llbracket \mathrm{VP} 1 \rrbracket^{g, h}=\left\{\lambda y \cdot \lambda w . \operatorname{attended}_{w}(y, x) \mid x \in \llbracket\right.$ shenme huodong $\left.\rrbracket^{g}\right\}$

b. $\llbracket \mathrm{VP} 2 \rrbracket^{g}=\llbracket z h i \rrbracket^{g}\left(\llbracket \mathrm{VP} 1 \rrbracket^{g, h}\right)\left(\llbracket \mathrm{VP} 1 \rrbracket^{g}\right)$

$=\left\{\lambda y . \lambda w . \forall P\left[P_{w}(y) \rightarrow \lambda w^{\prime}\right.\right.$. attended $\left._{w^{\prime}}(y, x) \subseteq P(y)\right] \mid x \in \llbracket$ shenme huodong $\left.\rrbracket^{g}\right\}$

$\approx\left\{\lambda y \cdot \lambda w . y\right.$ attended $_{w}$ nothing other than $x \mid x \in \llbracket$ shenme huodong $\left.\rrbracket^{g}\right\}$

c. $\llbracket \mathrm{IP} \rrbracket^{g} \approx\left\{\lambda w\right.$. Libai attended ${ }_{w}$ nothing other than $x \mid x \in \llbracket$ shenme huodong $\left.\rrbracket^{g}\right\}$

Now we have seen how focus intervention follows from the quantificational structure of focus-sensitive operators, rather than merely from the presence of focussensitive operators. It leads to the understanding that a focus-sensitive operator in a $w h$-question is not an intervener in and of itself. Focus intervention in $w h$-questions is not just caused by focus interveners, but by a conspiracy of focused phrases, $w h$-phrases, and the quantificational nature of focus-sensitive operators.

However, this is not the only thing the quantificational domain approach buys us. The approach also predicts that focus intervention is likely a phenomenon independent of $w h$-questions. If what matters is the interaction of ordinary alternatives and focus alternatives in the scope of focus-sensitive operators, then we should expect focus intervention with any expressions that evoke ordinary alternatives. The following two sections take up this expectation in greater detail.

\section{Focus intervention with non-interrogative $w h$-phrases}

According to Kratzer \& Shimoyama (2002) and Shimoyama (2006), Hamblin's semantics can be extended to non-interrogative $w h$-phrases. They assume that a $w h$-phrase uniformly denotes a set of alternatives as its ordinary-semantic value and undergoes set expansion. Whether it gives rise to an interrogative or a noninterrogative reading depends on the operator that closes the set expansion.

There are two non-interrogative uses of $w h$-phrases in Mandarin. One can be found in the restrictor of (wunlun)...dou, where the wh-phrase is interpreted universally (Cheng 1991, Lin 1996); another can be found in non-veridical contexts, such as in the scope of epistemic modals, where the $w h$-phrase is interpreted as an existential indefinite (Lin 1998, Liao 2011). We take up these two uses one by one.

An instance of a wh-phrase in the restrictor of (wunlun)...dou can be seen in (9), which is a $w h$-unconditional. The sentence expresses that the resolution of the issue described by the wulun-adjunct is independent of the truth of the main clause (see also Rawlins 2008, 2013). 
(9) [IP3 Wulun [IP1 Libai yaoqing shei], [IP2 wo dou bu hui chuxi no.matter Libai invite who, I DOU not will attend wanyan]].

dinner

'No matter who Libai invites, I will not attend the dinner.'

The LF structure of (9) is given in (11) and the semantic composition is given in (11).

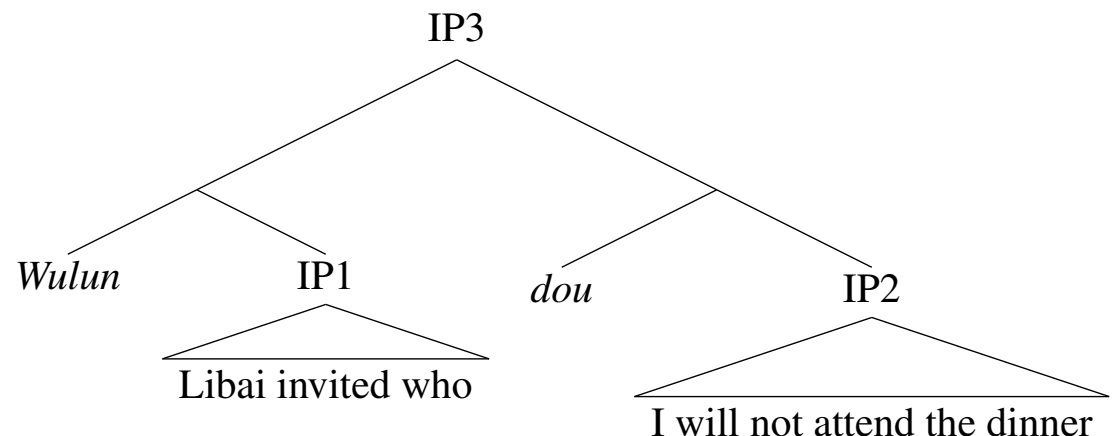

(11) a. $\llbracket$ shei $\rrbracket^{g}=\{$ John, Mary $\}$

b. $\llbracket \mathrm{IP} 1 \rrbracket^{g}=\left\{\lambda w\right.$. invites $_{w}($ Libai, $x) \mid x \in \llbracket$ shei $\left.\rrbracket^{g}\right\}$

c. $\llbracket$ wulun $\alpha$ dou $\beta \rrbracket^{g}=\lambda w$. $\forall p \in \llbracket \alpha \rrbracket^{g}\left[p(w) \rightarrow \llbracket \beta \rrbracket^{g}(w)\right]$

d. $\llbracket \mathrm{IP} 2 \rrbracket^{g}=\lambda w . \forall p \in \llbracket \mathrm{IP} 1 \rrbracket^{g}\left[p(w) \rightarrow \neg\right.$ will-attend $_{w}(\mathrm{I}$, the dinner $\left.)\right]$

Clearly, the computation of the $w h$-clause is similar to that of $w h$-questions. The $w h$-phrase denotes a set of individuals, which keeps expanding with the help of pointwise functional application until it is selected by the operator (wulun)...dou as its domain restriction. ${ }^{2}$

Accordingly, the quantificational domain approach predicts focus intervention in the $w h$-clause of a $w h$-unconditional if the right configuration results. The expectation is borne out by the Mandarin sentences in (12).

a. ?*Wulun ta zhi yaoqing-le Libai ${ }_{F}$ chuxi shenme huodong, wo no.matter he only invite-Asp Libai attend what activity I dou hui daochang.

DOU will go

Intended 'No matter which activity he only invited Libai ${ }_{F}$ to attend, I will go.'

2 We assume, for the sake of simplicity, that (wulun)...duo is a complex, discontinuous operator. 
Generalized focus intervention

b. ?* Wulun zhiyou Libai ${ }_{F}$ yaoqing-le shei canjia wanyan, wo dou no.matter only Libai invite-Asp who attend dinner I DOU hui daochang.

will go

Intended 'No matter who only Libai $F$ invites to attend the dinner, I will go.'

Moreover, it correctly predicts that the $w h$-phrase in a wh-unconditional can associate with a focus-sensitive operator without giving rise to focus intervention:

(13) a. Wulun Libai zhi yaoqing-le shei chuxi wanyan, wo dou hui no.matter Libai only invite-Asp who attend dinner I DOU will daochang.

go

'No matter who is the person $x$ such that Libai invites nobody other than $x$, I will go.'

b. Wulun zuizhong zhiyou shei neng chuxi wanyan, wo dou hui no.matter at.last only who can attend dinner I DOU will daochang.

go

'No matter who is the person $x$ such that nobody other than $x$ can attend the dinner at last, I will go.'

By contrast, when a focus-sensitive operator is outside a wulun-adjunct, it should not give rise to focus intervention, hence the well-formedness of (14a); nor should it be able to associate with the $w h$-phrase, hence the ill-formedness of (14b). This is because the set of alternatives denoted by the $w h$-phrase is no longer available when it is closed by (wulun) ... dou.

(14) a. Zhiyou Libai ${ }_{F}$ wulun chuxi shenme huodong dou hui chuan only Libai no.matter attend what activity DOU will wear xizhuang.

suit

'Libai is the only person who will wear a suit, no matter which activity he attends.'

b. * Libai zhiyou wulun chuxi shenme huodong dou hui chuan Libai only no.matter attend what activity DOU will wear xizhuang.

suit 
Now, we can turn to non-interrogative wh-phrases interpreted as existential indefinites. Lin (1998) has reported a series of licensing conditions for this type of $w h$-phrases. Liao (2011) has further generalized them to non-veridical contexts. The following example shows the existential reading of a $w h$-indefinite in the scope of an epistemic modal.

(15) Keneng Libai chi-le shenme dongxi. possibly Libai eat-Asp what thing

'It is possible that Libai ate something.'

According to Kratzer \& Shimoyama (2002), the modal is combined with an existential closure, which closes the set expansion of a $w h$-phrase, allowing the $w h$-phrase to receive an existential interpretation. Following Reinhart 2006 and Dong 2009, moreover, we assume that the existential closure can freely apply to VP or IP (see also Chierchia 2001; Lin 2004). The definitions of the existential closure (based on Kratzer \& Shimoyama 2002: 7) at the VP (predicate) level and at the IP (proposition) level are given below:

a. Predicate-level existential closure For $\llbracket \alpha \rrbracket^{g} \subseteq D_{<e, s t>}, \llbracket \exists \alpha \rrbracket^{g}=\lambda y . \lambda w . \exists P \in \llbracket \alpha \rrbracket^{g} \wedge P_{w}(y)$

b. Proposition-level existential closure For $\llbracket \alpha \rrbracket^{g} \subseteq \mathrm{D}_{<s t>}, \llbracket \exists \alpha \rrbracket^{g}=\lambda w . \exists p \in \llbracket \alpha \rrbracket^{g} \wedge p(w)$

We assume that the proposition-level existential closure is used in (15), whose LF structure and semantic composition are (17) and (18).

[IP3 Keneng [IP2 $\exists$ [IP1 Libai chi-le shenme dongxi]]] possibly Libai eat-Asp what thing

a. $\llbracket \operatorname{IP} 1 \rrbracket^{g}=\left\{\lambda w\right.$. ate ${ }_{w}($ Libai, $x) \mid x \in \llbracket$ shenme dongxi $\left.\rrbracket^{g}\right\}$

b. $\llbracket \mathrm{IP} 2 \rrbracket^{g}=\lambda w . \exists p \in \llbracket \mathrm{IP} 1 \rrbracket^{g} \wedge p(w)$

c. $\llbracket \mathrm{IP} 3 \rrbracket^{g}=\lambda w . \exists w^{\prime}\left[w^{\prime} \in \xi_{w} \wedge p\left(w^{\prime}\right)\right]$, where $\xi$ is the set of worlds epistemically accessible from $w$

Lin (2004) points out that an existential $w h$-indefinite may interact with other scope-bearing elements. For example, in (19a), the existential $w h$-phrase can take narrow or wide scope relative to negation, giving rise to the readings in (19b) and (19c), respectively.

(19) a. Keneng Libai mei zuo shenme cai. possibly Libai not cook what dish

b. 'It is possible that Libai didn't cook anything.' 
Generalized focus intervention

c. 'It is possible that there is something that Libai didn't cook.'

The two readings can be obtained by applying the existential closure below or above negation, as shown in the two representations in (20).

a. [possibly Libai not [ $\exists$ [cook what dish ]]]

b. [possibly $[\exists$ [Libai not cook what dish]]]

Interestingly, when the subject associates with a focus-sensitive operator, as in (21a), only the narrow scope reading of the existential $w h$-phrase (21b) is available. The wide scope reading (21c) is blocked.

a. Keneng zhiyou Libai ${ }_{F}$ mei zuo shenme cai. possibly only Libai not cook what dish

b. [possibly only $\operatorname{Libai}_{F}[\exists$ [not cook what dish]]] $\approx$ It is possible that nobody other than Libai cooked any dish.

c. ?* [possibly [ $\exists$ [only Libai $F$ not cook what dish]]] $\approx$ It is possible that there is some dish that nobody other than Libai cooked.

The unavailability of the wide scope reading falls under the prediction of the quantificational domain approach: in (21c), the focus-sensitive operator scopes over the focus alternatives evoked by the focused phrase and the ordinary alternatives evoked by the $w h$-phrase. The interaction of these two types of alternatives gives rise to an inappropriate quantificational domain for the focus-sensitive operator, resulting in focus intervention. By contrast, in (21b), the set expansion of the wh-phrase has been closed by $\exists$ before the focus-sensitive operator is composed. There is no interaction of the ordinary alternatives and the focus alternatives. As a consequence, focus intervention does not arise.

The same contrast also surfaces in the restrictor of a conditional. The existential wh-phrase in (22a) can take narrow or wide scope relative to the negation.

a. Yaoshi ni bu xiang chi shenme de-hua, qing tiqian rang wo if you not want eat what if please beforehand let me zhidao.

know

b. [ [if you not $\exists$ [want to eat what]] ...]

$\approx$ If you don't want to eat anything, please let me know in advance.

c. [ [if $\exists$ [you not want to eat what]] ... ]

$\approx$ If there is something that you don't want to eat, please let me know in advance. 
However, replacing the subject with a focused phrase associated with a focussensitive operator blocks the wide scope reading. Hence, (23a) can only mean (23b), but not (23c).

(23) a. Yaoshi zhiyou Libai $F$ bu xiang chi shenme de-hua, qing

if only Libai not want eat what if please

tiqian rang wo zhidao.

beforehand let me know

b. 'If only Libai ${ }_{F}$ doesn't want to eat anything, please let me know in advance.' (if $>$ not $>$ wh)

c. ?* 'If there is something that only $\operatorname{Libai}_{F}$ doesn't want to eat, please let me know in advance.' (if $>w h>n o t)$

Interrogative and non-interrogative $w h$-phrases appear in different linguistic contexts and are licensed by different operators. The fact that they pattern uniformly with respect to focus intervention reveals that focus intervention has nothing to do with the type of operators that license these wh-phrases. In fact, in what follows, we show that it also does not matter if the expressions that participate in focus intervention are wh-phrases or not.

\section{Focus intervention with disjunctive phrases}

Recent studies have also extended Hamblin's semantics to disjunction (Aloni 2003, Simons 2005, Alonso-Ovalle 2006). ${ }^{3}$ According to this approach, a disjunctive phrase has the following denotation:

$$
\llbracket \mathrm{A} \text { or } \mathrm{B} \rrbracket^{g}=\{\mathrm{A}, \mathrm{B}\}
$$

If this view is correct, a disjunctive phrase is just like a wh-phrase, denoting a set of (two or more) alternatives as its ordinary-semantic value. Consequently, the quantificational domain approach predicts that focus intervention should surface in sentences with disjunctive phrases in the same environments that trigger focus intervention in wh-questions. In the following subsections, we show that the prediction is borne out by disjunctive phrases in declarative sentences and those in questions.

\subsection{Declarative disjunctive sentences}

Partee \& Rooth (1983) have argued that disjunctions are scope-bearing elements (see also Larson 1985, Simons 2005, a.o.), which may participate in scope interactions.

3 We thank Simon Charlow for drawing our attention to the issue of disjunction. 
For example, (25a) has two de dicto readings depending on the relative scope of the disjunction and the intensional verb look for. The disjunction is said to take narrow scope in (25b) and wide scope in (25c). The wide scope reading of the disjunction can be forced by a continuation like But I'm not sure which. ${ }^{4}$

a. Mary is looking for [Disjp a maid or a cook].

b. Mary is looking for $x, x$ is a maid or $x$ is a cook

c. Mary is looking for a maid or Mary is looking for a cook.

Interestingly, the wide scope reading of disjunction becomes unavailable when a focus-sensitive operator and its associate precede a disjunction. Consider the examples in (26). (26a) has no focus-sensitive operator or focus associate, hence, the disjunction can take wide scope, as evidenced by the felicity of the continuation. However, no continuation is possible in (26b-c), suggesting that the presence of the focus-sensitive operator and its associate blocks the wide scope reading of the disjunction.

(26) a. Peter introduced John to [DisjP Mary or Sue]. But I'm not sure which.

b. Peter only introduced [John $]_{F}$ to [Disjp Mary or Sue]. ?*But I'm not sure which.

c. Only $[\text { Peter }]_{F}$ introduced John to [Disjp Mary or Sue]. ?*But I'm not sure which.

The same phenomenon is also found in Mandarin, only in a more remarkable way. To begin with, Crain (2012: 240) has reported that disjunction in Mandarin necessarily takes wide scope relative to negation.

$$
\begin{aligned}
& \text { Yuehan meiyou chi [Disjp pingguo huozhe li]. } \\
& \text { John not eat apple or pear } \\
& \text { 'John didn't eat apples or he didn't eat pears.' }
\end{aligned}
$$

However, when a focus-sensitive operator and its associate precede the disjunctive phrase, as in (28a), the disjunction can only take narrow scope, i.e., (28b), but not wide scope, i.e., (28c) (Crain 2012: 242-243).

\section{a. Zhiyou Yuehan $F$ chi-le [Disjp pingguo huozhe li].}

$$
\text { only John eat-Asp apple or pear }
$$

4 The sentence also has a de re reading, which asserts the existence of a particular maid and a particular cook. It seems to us that such a reading requires both the disjunction and the disjuncts (i.e., the indefinite noun phrases) to take wide scope. How indefinite noun phrases behave in focus intervention contexts is an intriguing issue. However, we must leave it for another occasion. 
b. 'John is the only person who ate any apple or pear.' (only- $\mathrm{XP}_{F}>$ or)

c. ?* 'Only John ate any apple or only John ate any pear.' $\left(\right.$ or $>$ only- $\left.\mathrm{XP}_{F}\right)$

According to the quantificational domain approach, the unavailability of the wide scope reading is a result of focus intervention. Following Aloni 2003, Simons 2005 and Alonso-Ovalle 2006, a disjunctive phrase denotes a set of alternatives as its ordinary semantic value, just like an in-situ $w h$-phrase. Therefore, the wide scope reading of disjunction can be derived along the same lines as $w h$-in-situ questions. The only difference is that the former requires an existential closure (Kratzer \& Shimoyama 2002), as defined in (16a-b), to close the set expansion. We illustrate the LF of (26a) in (29a) and the steps of the composition in (29b-d).

We take the derivation of (26a) to illustrate the compositional semantics of a sentence with a disjunctive phrase:
a. [IP2 $\exists$ [IP1 Peter introduced John to [DisjP Mary or Sue ]]]
b. $\llbracket$ DisjP $\rrbracket^{g}=\{$ Mary, Sue $\}$
c. $\llbracket \mathrm{IP} 1 \rrbracket^{g}=\left\{\lambda w\right.$. introduce ${ }_{w}($ Peter, John, $x) \mid x \in \llbracket$ DisjP $\left.^{g} \rrbracket^{g}\right\}$
d. $\llbracket \mathrm{IP} 2 \rrbracket^{g}=\lambda w . \exists p \in \llbracket \mathrm{IP} 1 \rrbracket^{g} \wedge p(w)$

Let's turn to the examples in which the wide scope reading of disjunction is blocked. Consider the sentence in (26b), whose LF structure of the wide scope reading is given in (30). It is a typical focus intervention configuration: the focussensitive operator scopes over focus alternatives evoked by $J o h n_{F}$ and ordinary alternatives evokes by the disjunctive phrase. Consequently, focus intervention rules out the wide scope reading.

$\left[\exists\left[\right.\right.$ Peter [VP2 only [vP1 introduced John ${ }_{\mathrm{F} 1}$ to [DisjP Mary or Sue]]]]]

Although the wide scope reading of disjunction is prevented, the narrow scope reading is still available in (26b). This contrast follows straightforwardly from the quantificational domain approach. The relevant reading is represented by the LF structure in (31).

$$
\text { [IP Peter [VP3 only [VP2 } \exists\left[\mathrm{VP1} \text { introduced } \mathrm{John}_{F 1}\right. \text { to [Disjp Mary or Sue]]]] }
$$

In the above LF, the set of ordinary alternatives denoted by the disjunctive phrase is selected by the predicate-level $\exists$ and hence the set expansion is closed upon VP2, prior to the activation of the designated assignment function $h$. Therefore, there is no interaction between the ordinary alternatives evoked by the disjunctive phrase and the focus alternatives evoked by the focused phrase. The composition proceeds as follows: 
a. $\llbracket \mathrm{VP} 1 \rrbracket^{g}=\left\{\lambda y \cdot \lambda w\right.$. introduce $w(y$, John $\left.x) \mid x \in \llbracket \mathrm{DisjP} \rrbracket^{g}\right\}$

b. $\llbracket \mathrm{VP} 2 \rrbracket^{g}=\lambda y \cdot \lambda w . \exists P \in \llbracket \mathrm{VP} 1 \rrbracket^{g} \wedge P_{w}(y)$ $=\lambda y \cdot \lambda w$. $\operatorname{introduce}_{w}(y$, John, Mary $) \vee \operatorname{introduce}_{w}(y$, John, Sue $)$

c. $\llbracket \mathrm{VP} 2 \rrbracket^{g, h}=\lambda y . \lambda w$. introduce ${ }_{w}(y, h(1)$, Mary $) \vee \operatorname{introduce}_{w}(y, h(1)$, Sue $)$

d. $\llbracket \mathrm{VP} 2 \rrbracket^{f}=\left\{\llbracket \mathrm{VP} 2 \rrbracket^{g, h} \mid h \in \mathrm{H}\right\}$ $=\left\{\lambda y . \lambda w\right.$. introduce $_{w}(y, h(1)$, Mary $) \vee$ introduce $_{w}(y, h(1)$, Sue $\left.) \mid h \in \mathrm{H}\right\}$

e. $\llbracket \mathrm{VP} 3 \rrbracket^{g}=\lambda y \cdot \lambda w . \forall P \in \llbracket \mathrm{VP} 2 \rrbracket^{f}\left[P_{w}(y) \rightarrow \llbracket \mathrm{VP} 2 \rrbracket^{g}(y) \subseteq P(y)\right]$

Note that $\llbracket \mathrm{VP} 2 \rrbracket^{f}$ is not a set of sets of propositions but rather a set of propositions, as shown in (32d). As a consequence, $\llbracket \mathrm{VP} 2 \rrbracket^{f}$ is an appropriate quantificational domain for only. (32e) is a successful composition with no focus intervention.

For the sake of argument, the quantificational domain approach is not the only conceivable account that can explain the contrast between a wide scope disjunction and a narrow scope disjunction in the presence of a focus-sensitive operator. An account that treats a focus-sensitive operator as an inherent 'intervener' has the same effect. For example, Beck \& Kim's (2006) minimality account requires that the existential closure apply before a focus-sensitive operator to prevent the minimality effect triggered by the focus-sensitive operator; Han \& Romero's (2004a, 2004b) and Larson's (1985) account that the scope marker of a disjunctive phrase undergoes LF movement, when considered in conjunction with the assumption that a focussensitive marker is a LF barrier (Beck 1996), would offer similar explanatory power.

However, it is very difficult for these accounts to explain why the wide scope reading returns when a focus-sensitive operator takes the disjunction as its associate. Consider the examples in (33a-b). The felicity of the continuations indicate that the wide scope reading of the disjunction is available in these sentences.

a. Peter only introduced John to [Disjp Mary or Sue $]_{F}$. But I'm not sure which one.

b. Peter only introduced [DisjP John or Paul $]_{F}$ to Mary. But I'm not sure which one.

The same pattern is also observed in Mandarin. In (34), the focus-sensitive operator is associated with the disjunctive phrase. Here, the disjunction can have a wide scope reading.

Yuehan zhi chi-le [Disjp pingguo huozhe $l i]_{F}$.

John only eat-Asp apple or pear

'John ate nothing other than an apple or John ate nothing other than a pear.'

If the focus-sensitive operators in the above examples are indeed 'interveners' or 'barriers' at LF, the wide scope reading of the disjunctions remains mysterious. 
The mystery is resolved by the quantificational domain approach in a principled way. Take (33a) as an example. The LF structure in (35), in which the existential closure is introduced above only, represents the wide scope reading of the disjunction. We assume that the disjunctive phrase bears a focus index, which is inherited from its disjuncts through percolation (see also Beck \& Kim 2006; Truckenbrodt 2013). Hence, the designated assignment function $h$ is activated, giving rise to focus alternatives, as shown in (36).

$$
\text { [IP2 } \left.\left.\left.\left.\exists[\text { IP1 Peter [VP2 only [vP1 introduced John to [DisjP Mary or Sue }]_{F 1}\right]\right]\right]\right]
$$

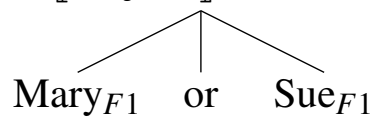

Note that the focus index of DisjP is the same as that of the disjuncts. As a result, $h$ maps a variable onto an object of the same type as the disjuncts (the assignment of the focus index will be taken up again in section 5). Based on this assumption, we can compute the LF structure in (35) following the steps shown in (37). The result is a wide scope reading for the disjunction.

a. $\llbracket \mathrm{VP} 1 \rrbracket^{g}=\left\{\lambda y \cdot \lambda w\right.$. introduce $_{w}(y$, John, $x) \mid x \in \llbracket$ DisjP $\left.\rrbracket^{g}\right\}$

b. $\llbracket \mathrm{VP} 1 \rrbracket^{g, h}=\lambda y \cdot \lambda w$. introduce ${ }_{w}(y$, John $h(1))$

c. $\llbracket \mathrm{VP} 1 \rrbracket^{f}=\left\{\lambda y \cdot \lambda w\right.$. introduce $_{w}(y$, John $\left.h(1)) \mid h \in \mathrm{H}\right\}$

d. $\llbracket \mathrm{VP} 2 \rrbracket^{g}=\llbracket$ only $\rrbracket^{g}\left(\llbracket \mathrm{VP} 1 \rrbracket^{f}\right)\left(\llbracket \mathrm{VP} 1 \rrbracket^{g}\right)$ $=\left\{\begin{array}{l}\lambda y \cdot \lambda w \cdot \forall P \in \llbracket \mathrm{VP} 1 \rrbracket^{f}\left[P_{w}(y) \rightarrow \lambda y \cdot \lambda w^{\prime} . \text { introduce }_{w^{\prime}}(y, \text { John, } x) \subseteq P(y)\right] \\ \mid x \in \llbracket \mathrm{Disj} \mathrm{P} \rrbracket^{g}\end{array}\right\}$ $\approx\left\{\begin{array}{l}\lambda y \cdot \lambda w \cdot y \text { introduce }_{w} \text { John to nobody other than Mary } \\ \lambda y \cdot \lambda w \cdot y \text { introduce }_{w} \text { John to nobody other than Sue }\end{array}\right\}$

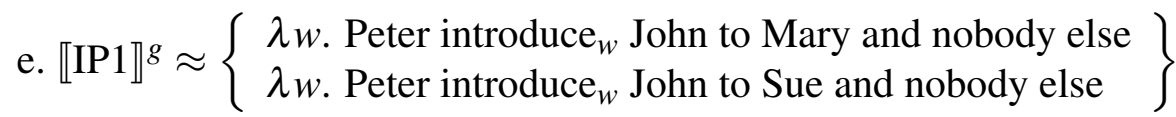

f. $\llbracket \mathrm{IP} 2 \rrbracket^{g}=\lambda w . \exists p \in \llbracket \mathrm{IP} 1 \rrbracket^{g} \wedge p(w)$

In short, disjunctive phrases in declarative sentences pattern like (non-)interrogative $w h$-phrases in the contexts of focus intervention. The parallelism is not surprising given the assumption we have adopted, namely, that disjunctive phrases and whphrases share the same type of ordinary semantic values, i.e., sets of alternatives. In the next section, we will turn to alternative questions, another structure involving disjunction, in which focus intervention presents itself consistently. 
Generalized focus intervention

\subsection{Alternative questions}

Disjunctive phrases occur not only in declarative sentences but also in questions, giving rise to alternative questions. Adopting the view that disjunctive phrases denote sets of alternatives, a lot of studies have proposed that the compositional analysis of alternative questions follows Hamblin's semantics (von Stechow 1991; Biezma \& Rawlins 2012; see also Beck \& Kim 2006 ${ }^{5}$ ). Specifically, the disjunctive phrase in (38a) denotes a set of the ordinary semantic values of its disjuncts, i.e., a set of properties, as in (38b). With the help of pointwise functional application, the interpretation of the alternative question is given in (38c); that is, it denotes a set of propositions.

(38) a. [CP Did John [DisjP dance or sing]]?

b. $\llbracket$ DisjP $\rrbracket^{g}=\left\{\lambda x . \lambda w\right.$. danced $\left.{ }_{w}(x), \lambda x . \lambda w . \operatorname{sang}_{w}(x)\right\}$

c. $\llbracket \mathrm{CP} \rrbracket^{g}=\left\{\lambda w . \operatorname{danced}_{w}(\mathrm{John}), \lambda w . \operatorname{sang}_{w}(\mathrm{John})\right\}$

If focus intervention can be found in declaratives with disjunctions, it is expected to show up in alternative questions.

According to Beck \& Kim 2006, focus intervention does show up in alternative questions. Consider some of their examples in (39a-b) and (40a-b) (taken from Beck $\&$ Kim 2006: 172; the notations are our own).
a. ?*Did only Mary $F$ introduce Sue [Disjp to Bill or (to) Tom]?
b. ?*Did only Mary ${ }_{F}$ introduce [Disjp Sue or Molly] to Bill?

More examples show that the choice of focus-sensitive operators and their syntactic positions (pre-IP or pre-VP) do not affect the generalization, as long as the focussensitive operators precede two different types of sets of alternatives:

a. ?*Did Peter only give Mary $F$ [Disjp a book or a pen]?

b. ?*Did Peter also give Mary $F[$ DisjP a book or a pen]?

We have already known from the last section that the quantificational domain approach expects the disjunctive phrases in (39) and (40) to take narrow scope since the wide scope reading is ruled out by focus intervention. Unfortunately, for an alternative question to be well formed, the disjunctive phrase must take wide scope (Larson 1985; Han \& Romero 2004a; Biezma \& Rawlins 2012). As a consequence, these sentences cannot be interpreted as alternative questions. The only available

5 Beck \& Kim (2006) propose that disjunctive phrases denote sets of alternatives as their focus semantic values rather than ordinary semantic values in alternative questions. This is different from the standard version of Hamblin's semantics. We will not compare them in this paper. 
interpretations are yes/no-questions, which require the disjunctive phrases to take narrow scope.

Although Beck \& Kim (2006) came to the same conclusion based on their minimality account, the quantificational domain approach makes a prediction that is crucially different from theirs. According to Beck \& Kim 2006, focus-sensitive operators interfere with the disjunctive phrases of alternative questions. As a result, the sentences in (41) should be ruled out, contrary to fact.

a. Did Peter only give $[\text { DisjP Mary or Jane }]_{F}$ a pen?

b. Did Peter also give [Disjp Mary or Jane $]_{F}$ a pen?

However, the quantificational domain approach predicts that these sentences should be acceptable. As argued in the last section, a disjunctive phrase can associate with a focus-sensitive operator and take wide scope at the same time. So, they can give rise to alternative questions like the ones in (41).

He (2011) argued that alternative questions in Mandarin should be analyzed along the lines of Hamblin's semantics (see also Erlewine to appear). If this is indeed the case, the contrast between (39-40) and (41) should also be found in Mandarin. We confirm the contrast with the following set of data, Here, haishi introduces disjuncts almost exclusively for alternative questions.

a. ?* Zhiyou [Libai $]_{F}$ he-le [Disjp kafei haishi hongcha]?
only Libai drink-Asp coffee or black.tea Intended 'Which one of $\mathrm{x}, \mathrm{x}$ is coffee or tea ,such that only Libai drank $\mathrm{x}$ ?'

b. Libai zhi he-le [Disjp kafei haishi hongcha]?

Libai only drink-Asp coffee or black.tea

'Which one of $x, x$ is coffee or tea, such that Libai only drank $x$ ?'

Although focus intervention in alternative questions is not new in the literature, only now do we realize that focus-sensitive operators can take disjunctive phrases as their associates without triggering focus intervention. This empirical advancement is a natural consequence of the theoretical progress introduced by the quantificational domain approach to focus intervention.

\section{The availability of the focus index}

An astute reader may have noticed that the proposed analysis requires a non-uniform assumption on the availability of focus indices in $w h$-phrases and disjunctive phrases. In particular, $w h$-phrases are assumed not to bear any focus index, whether or not they are interpreted as focus. However, disjunctive phrases can come with or without 
focus indices, depending on whether they are interpreted as focus or not. Does this non-uniform assumption weaken the parallelism between $w h$-phrases and disjunctive phrases? We argue that it does not, since we have independent reasons to believe that the difference is needed.

The first consideration is a theoretical argument related to feature redundancy. As pointed out by Watanabe (2002) and Ishihara (2003), in-situ interrogative $w h$ phrases should not share the same focus feature with focused phrases, even though the former are always phonetically prominent in questions. The reason is that $w h$ phrases inherently denote sets of alternatives: if they were assigned a focus feature that evokes alternatives, it would result in redundancy. By contrast, denoting sets of alternatives is not an inherent property of other linguistic items, hence a focus feature must be assigned to evoke their alternatives. Since a disjunctive phrase is made up of two or more non-wh-expressions, assigning a focus feature in the form of an index to the individual disjuncts does not result in redundancy. The only extra stipulation we need is to allow the focus index on the disjuncts to become the focus index of the disjunctive phrase. We have implemented this idea using the notion of feature percolation.

The second reason is an empirical argument coming from association with focus. Since (for reasons unknown to us) English does not readily allow focus association with wh-phrases, we use Mandarin examples to illustrate this point. Consider the following sentences:
a. Libai zhi chi-le shenme dongxi?
Libai only ate-Asp what thing
'What is the thing $\mathrm{x}$ such that Libai only ate $\mathrm{x}$ ?'
Libai only ate-Asp rice or noodles
'Did Libai only ate rice or noodles?'
b. Libai zhi chi-le mifan haishi miantiao?

For concreteness, let us assume that the wh-phrase in (43a) denotes a set of contextually salient food:

$$
\llbracket \text { shenme dongxi } \rrbracket^{g}=\{\text { vegetables, rice, noodles, ... }\}
$$

Since the wh-phrase bears no focus index, the focus-sensitive operator takes its secondary value, which is identical to its ordinary-semantic value, as the quantificational domain. Hence, if the answer to (43a) is 'rice', it implies that Libai didn't eat vegetables, noodles or any other contextually salient food.

What about the denotation of the disjunctive phrase? We have assumed that the ordinary-semantic value of a disjunctive phrase is a set of alternatives made of its disjuncts. In this case, the denotation of the disjunctive phrase in (43b) is as follows: 


$$
\llbracket \text { mifan haishi miantiao } \rrbracket^{g}=\{\text { rice, noodles }\}
$$

If a disjunctive phrase has no focus index even when it is interpreted as a focus, the focus-sensitive operator would make use of its secondary value, which is again identical to its ordinary-semantic value, as the quantificational domain. Then, the same answer 'rice' should only imply that Libai didn't eat noodles. In other words, Libai is free to eat anything else not included in this domain. However, the domain of the focus-sensitive operator in (43b) is larger than (45). For example, the answer 'rice' requires that Libai only ate rice, but not noodles or anything else. What this tells us is that when the disjunctive phrase is a focus, the exhaustivity holds true of a wider domain. We argue that the focus index on a disjunctive phrase, which is inherited from the disjuncts (see section 4.1), gives rise to a focus-semantic value that contains at least the members denoted by the ordinary-semantic value of the disjunctive phrase.

\section{Conclusion}

The quantificational domain approach is one way of cashing out Beck's (2006) insight that focus intervention can be studied in terms of alternative semantics. Our implementation of the neo-Hamblin framework relies on the intuition that focus alternatives and ordinary alternatives are alternatives along different dimensions. The interaction of different types of alternatives gives rise to inappropriate quantificational domains of focus-sensitive operators. In the absence of focus alternatives or ordinary alternatives, the presence of focus-sensitive operators does not cause any harm.

This approach leads to a number of consequences. First, focus-sensitive operators are no longer taken to be inherent interveners; they become problematic if they take a set of sets of alternatives as its quantificational domain. On this view, focus intervention is not an intervention phenomenon, but a purely quantificational phenomenon. Second, this approach allows for a very general understanding of focus intervention, providing a unified analysis for focus intervention with interrogative wh-phrases, non-interrogative $w h$-phrases and disjunctive phrases.

\section{References}

Aloni, Maria. 2003. Free choice in modal contexts. In Matthias Weisgerber (ed.), Proceedings of Sinn und Bedeutung 7, 25 - 37. Konstanz: Universitat Konstanz. Alonso-Ovalle, Luis. 2006. Disjunction in alternative semantics: University of Massachusetts at Amherst dissertation.

Beck, Sigrid. 1996. Quantified structures as barriers for LF movement. Natural Language Semantics 4. 1-56. 
Generalized focus intervention

Beck, Sigrid. 2006. Intervention effects follow from focus interpretation. Natural Language Semantics 14. 1-56.

Beck, Sigrid \& Shin-Shook Kim. 2006. Intervention effects in alternative questions. Journal of Comparative German Linguistics 9. 165-208.

Biezma, Maria \& Kyle Rawlins. 2012. Responding to alternative and polar questions. Linguistics and Philosophy 35. 361-406.

Cable, Seth. 2010. The grammar of $Q$. Oxford: Oxford University Press.

Cheng, Lisa Lai-Shen. 1991. On the typology of WH-questions: Massachusetts Institute of Technology dissertation.

Chierchia, Gennaro. 2001. A puzzle about indefinites. In Carlo Cecchetto, Gennaro Chierchia \& Maria Teresa Guasti (eds.), Semantic Interfaces: Reference, Anaphora and Aspect, 51-89. Stanford, California: CSLI.

Crain, Stephen. 2012. The Emergence of Meaning. Cambridge: Cambridge University Press.

Dong, Hongyuan. 2009. Issues in the semantics of Mandarin questions: Cornell University dissertation.

Erlewine, Michael Y. to appear. Alternative questions through focus alternatives in Mandarin Chinese. In Proceedings of Chicago Linguistics Society 48, .

Hamblin, Charles L. 1973. Questions in Montague English. Foundations of Language 10. 41-53.

Han, Chung-hye \& Maribel Romero. 2004a. Disjunction, focus, and scope. Linguistic Inquiry 35(2). 179-217.

Han, Chung-hye \& Maribel Romero. 2004b. The syntax of Whether/Q...or questions: Ellipsis combined with movement. Natural Language and Linguistic Theory 22(3). 527-564.

He, Chuansheng. 2011. Expansion and closure: towards a theory of wh-construals in Chinese: The Hong Kong Polytechnic University dissertation.

Ishihara, Shinichiro. 2003. Intonation and interface conditions: Massachusetts Institute of Technology dissertation.

Kotek, Hadas \& Michael Y. Erlewine. to appear. Covert pied-piping in English multiple wh-questions. Linguistic Inquiry .

Kratzer, Angelika. 1991. The representation of focus. In Arnim von Stechow \& Dieter Wunderlich (eds.), Semantics: An International Handbook of Contemporary Research, 825-834. Berlin: de Gruyter.

Kratzer, Angelika \& Junko Shimoyama. 2002. Indeterminate pronouns: The view from japanese. In Yokio Otsu (ed.), The Proceedings of the Third Tokyo Conference on Psycholinguistics, 1-25. Tokyo: Hituzi Syobo.

Larson, Richard K. 1985. On the syntax of disjunction scope. Natural Language and Linguistic Theory 3. 217-264.

Li, Haoze \& Jess Law. 2014. Focus intervention: a quantificational domain approach. 
In Leland Kusmer \& Jyoti Iyer (eds.), North-Eastern Linguistic Society (NELS) 44, 273-287. Amherst, MA: GLSA.

Liao, Hsiu-Chen. 2011. Alternatives and exhaustification: Non-interrogative uses of Chinese wh-words: Harvard University dissertation.

Lin, Jo-Wang. 1996. Polarity licensing and wh-phrase quantification in Chinese: University of Massachusetts dissertation.

Lin, Jo-Wang. 1998. On existential polarity wh-phrases in Chinese. Journal of East Asian Linguistics 7. 219-255.

Lin, Jo-Wang. 2004. Choice function and scope of existential polarity wh-phrases in Mandarin Chinese. Linguistics and Philosophy 27. 451-491.

Partee, Barbara Hall \& Mats Rooth. 1983. Generalized conjunction and type ambiguity. In R. Bäuerle, C. Schwarze \& Arnim von Stechow (eds.), Meaning, Use and Interpretation of Language, 362-383. Berlin: de Gruyter.

Rawlins, Kyle. 2008. (Un)conditionals: An investigation in the syntax and semantics of conditional structures: UCSC dissertation.

Rawlins, Kyle. 2013. (un)conditionals. Natural Language Semantics 40. 111-178.

Reinhart, Tanya. 2006. Interface Strategies. MIT Press.

Rooth, Mats. 1985. Association with focus: UMass dissertation.

Rooth, Mats. 1992. A theory of focus interpretation. Natural Language Semantics 1. 75-116.

Shimoyama, Junko. 2006. Indeterminate phrase quantification in Japanese. Natural Language Semantics 14. 139-173.

Simons, Mandy. 2005. Dividing things up: The semantics of or and the modal/or interactions. Natural Language Semantics 13. 271-316.

von Stechow, Arnim. 1991. Focusing and background operators. In Werner Abraham (ed.), Discourse Particles: Descriptive and Theoretical Investigations on the Logical, Syntactic and Pragmatic Properties of Discourse Particles in German, 37-81. Amsterdam, Philadelphia: Benjamins.

Truckenbrodt, Hubert. 2013. An analysis of prosodic F-effects in interrogatives: Prosody, syntax and semantics. Lingua 124. 131-175.

Watanabe, Akira. 2002. Loss of overt wh-movement in Old Japanese. In David Lightfoot (ed.), Syntactic Effects of Morphological Change, 179 - 195. New York: Oxford University Press.

Yatsushiro, Kazuko. 2009. The distribution of quantificational suffixes in Japanese. Natural Language Semantics 17. 141-173. 
Generalized focus intervention

Haoze Li

Department of Linguistics

The Chinese University of Hong Kong

haozeli@cuhk.edu.hk

Jess Law

Department of Linguistics

Rutgers, the State University of New Jersey

jesshk.law@rutgers.edu 